\title{
El sistema constructivo de la quincha en zonas rurales del Norte de Mendoza (Argentina)
}

\author{
The construction system of wattle and daub in \\ rural areas of the North of Mendoza (Argentina)
}

Autores:

Matías J. Esteves * mesteves@mendoza-conicet.gob.ar

Guadalupe Cuitiño** gcuitino@mendoza-conicet.gob.ar

*Instituto de Ciencias Humanas, Sociales y Ambientales, CONICET; Facultad de Arquitectura, Urbanismo y Diseño.

Universidad de Mendoza; Facultad de Ingeniería, Universidad Nacional de Cuyo, **Facultad de Ciencias Aplicadas a la Industria, Universidad Nacional de Cuyo, CONICET; Facultad de Arquitectura, Urbanismo y Diseño, Universidad de Mendoza

Argentina

\section{Resumen}

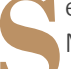

e analiza el sistema constructivo de la quincha en el norte de la provincia de Mendoza (Argentina) para luego reparar en la arquitectura vernácula como patrimonio, considerándola como parte del acervo histórico de la cultura y en relación con la identidad local. Para ello, se hace foco en dos zonas rurales del norte de la provincia en el contexto de tierras secas, para indagar respecto a sus posibles matices o variaciones y de esta forma contribuir a la diversidad del patrimonio vernáculo construido con tierra. La metodología que se utiliza responde principalmente al uso de técnicas cualitativas, las cuales involucran observación directa y entrevistas en profundidad a los habitantes locales. Los resultados muestran que la quincha presenta variaciones dependiendo del contexto natural y cultural en que se genera. Las conclusiones señalan la importancia de reconocer las diferencias que presenta el sistema constructivo en cada zona, aportando datos que permitan gestionar su puesta en valor y conservación.

Palabras clave: arquitectura vernácula, entramados, patrimonio cultural, tierras secas.

\section{Abstract:}

It is analyzed the construction system of the wattle and daub in the north of the province of Mendoza (Argentina) and then repaired in the vernacular architecture as heritage, considering it as part of the historical heritage of culture and in relation to local identity. For this, we focus on two rural areas in the north of the province in the context of dry lands, to inquire about its possible variations and contribute to the understanding of the vernacular heritage built with mug and clay. The methodology used responds mainly to the use of qualitative techniques, which involve direct observation and in-depth interviews with local inhabitants. The results show that the wattle and daub have variations depending on the natural and cultural context in which it is generated. The conclusions indicate the importance of recognizing the differences presented by the construction system in each area, providing data that allow managing its value and conservation.

Keywords: vernacular architecture, frameworks, cultural heritage, drylands. 


\section{Introducción}

En arquitectura, las construcciones en las cuales predomina el uso de técnicas constructivas con materiales naturales se denomina vernácula. Este concepto surge a mediados del siglo XX para contener o referenciar a la producción arquitectónica pensada y producida por el usuario con su bagaje cultural, donde se incluye el aprovechamiento de la naturaleza en su materialización y funcionamiento. De esta forma se marca la diferencia con aquella proyectada desde el ámbito disciplinar (Tomasi, 2011). Varios autores afirman que la arquitectura vernácula se despliega como un sistema social y cultural complejo, que surge de la dualidad cultura-naturaleza (Tamayo, Malo y García, 2019; Sánchez y Jiménez, 2010; Tillería, 2010).

Por sus características y su amplio uso a escala global, el Consejo Internacional de Monumentos y Sitios-ICOMOS- reconoce a esta arquitectura como factible de ser patrimonio y en su Carta del Patrimonio Vernáculo Construido la define como "la expresión fundamental de la identidad de una comunidad, de sus relaciones con el territorio y al mismo tiempo, la expresión de la diversidad cultural del mundo" (ICOMOS, 1999, p.1). En la articulación de lo vernáculo con el patrimonio, se coincide con Canivell y Pastor (2019) cuando exponen que "si bien la arquitectura vernácula es uno de los testimonios más antiguos de la construcción del hábitat humano, su reconocimiento como bien patrimonial es relativamente reciente. Más jóvenes aún, son las acciones destinadas a la protección de estos bienes" (p. 134).

El uso de la tierra cruda se enlaza con las tradiciones locales de prácticamente todos los continentes, donde aún se continúan empleando (Rotondaro, 2018). Es decir, no solo hace referencia a la dimensión histórica de la arquitectura, sino que también está en relación a temas actuales, debido a su bajo impacto ecológico y suponer una alternativa válida para la reducción del déficit habitacional en medios periurbanos o rurales debido al aprovechamiento de los materiales naturales propios de cada lugar (Rufino, 2013; Salman, 2018; Lárraga, Aguilar, Reyes y Fortanelli, 2014).

En Argentina, la tierra cruda ha sido el material principal a partir del cual se ha materializado la arquitectura vernácula (Urquijo, 1972). Pero, a lo largo del tiempo, este material ha sido desplazado por otros de mayor resistencia, lo cual ha sido favorecido por los avances tecnológicos y la elaboración de códigos de edificación tales como la Norma INPRES CIRSOC en Argentina, sobre todo en zonas con actividad sísmica. A su vez, en gran parte del siglo XX, "la construcción con tierra cruda se asoció a la pobreza y el atraso. Se instaló una cultura que tendía a desprestigiar este tipo de arquitectura y negar sus valores ambientales" (Lacoste, Premat y Bulo, 2014, p.86).

En la provincia de Mendoza, si bien actualmente en las zonas urbanas ya no predomina el uso de la tierra cruda en la construcción, en las zonas rurales aún hoy se encuentran edificios construidos con este material, y diversas comunidades todavía recurren a la tierra para construir sus viviendas. A esto se suma que en la literatura en general a nivel provincial, se encuentra una clara tendencia hacia los estudios históricos y técnicos vinculados con el adobe, en consonancia con su mayor uso en un momento histórico en las zonas urbanas (Cirvini y Manzini, 2016; Cirvini, 2017). En menor medida, se encuentran trabajos centrados en rescatar las particularidades de la quincha como sistema constructivo, especialmente en zonas rurales.

La provincia de Mendoza se emplaza en el ecosistema de tierras secas, donde se encuentran dos subregiones: las zonas irrigadas -oasis- y las zonas no irrigadas. Cada una posee características propias vinculadas con la disponibilidad de bienes naturales, principalmente agua y suelo cultivable, que inciden a su vez en la mayor o menor presencia de materiales naturales que sean factibles de ser empleados en la construcción. Considerando que se trata de arquitectura vernácula fuertemente arraigada a las condiciones naturales y sociales del sitio de emplazamiento, es que resulta relevante el estudio comparativo de la quincha en estas dos zonas rurales. A su vez, ambas zonas se encuentran atravesando profundos cambios que inciden en la pérdida de la arquitectura vernácula. La zona no irrigada se enfrenta a graves procesos de desertificación (Torres, 2010) que afectan a las actividades productivas y la presencia de materiales naturales para ser empleados en e sitio. En la zona rural del oasis se está produciendo el crecimiento acelerado de la ciudad, que se emplaza sobre suelos históricamente agrícolas provocando la desaparición de la arquitectura típica de la zona (Scoones, 2018).

La hipótesis sostiene que el sistema constructivo de la quincha presenta diferentes adaptaciones en las zonas rurales de Mendoza, según la disponibilidad de bienes naturales, la mano de obra utilizada y la distancia a los centros urbanos, que incide en el mayor o menor uso de materiales naturales. Comprender las características del sistema de la quincha y sus variaciones permite enriquecer a la arquitectura vernácula como patrimonio local, rescatando sus particularidades. El objetivo del trabajo es caracterizar al sistema constructivo de la quincha en Mendoza, haciendo foco en dos sectores rurales de la zona norte de la provincia, para indagar respecto de sus posibles matices o variaciones y de esta forma aportar a la comprensión del patrimonio vernáculo construido con tierra. 


\section{Metodología}

En la presente investigación se utilizaron principalmente técnicas cualitativas aplicadas en dos casos de estudio. Para ello, seleccionamos como unidad de análisis a la vivienda, ya que "es el producto más característico del diseño vernacular y, por lo tanto, el más influenciado por la cultura" (Rapoport, 2003, p.37). También se recurrió al análisis de fuentes primarias de información para indagar en las características naturales del territorio, las actividades productivas que se desarrollan y en los cambios territoriales sucedidos en el tiempo.

La selección de dos casos de estudio se vincula con que la provincia de Mendoza se emplaza en el ecosistema de tierras secas, con clima árido y precipitaciones que no superan los $250 \mathrm{~mm}$ anuales. En este contexto, el agua se convierte en el elemento organizador del territorio, donde se configuran dos subregiones contrastantes entre sí de acuerdo a la apropiación y uso del agua (Grosso Cepparo, 2017). Por un lado, las tierras secas irrigadas (Figura 1), que son los territorios con mayor acceso al agua superficial, y por ende al riego, y con posibilidad de cultivo -oasis- donde se asienta el 95\% de la población. Por otro lado, las tierras secas no irrigadas, que poseen escasa presencia de agua superficial, haciendo imposible la práctica de la agricultura. Además, poseen baja densidad poblacional y economías de subsistencia (Montaña, Torres, Abraham, Torres y Pastor, 2005). En este escenario ambiental, para el primer caso fueron consideradas las construcciones con quincha de las tierras secas irrigadas, donde se trabajó con los distritos de Los Corralitos y La Primavera, del departamento de Guaymallén y el distrito de Rodeo del Medio, departamento de Maipú, que forman parte del Cinturón Verde Agrícola. En el segundo caso, fueron las viviendas correspondientes a las tierras secas no irrigadas, donde se trabajó con los distritos de Lagunas del Rosario y Asunción, en el departamento de Lavalle (Figura 1)

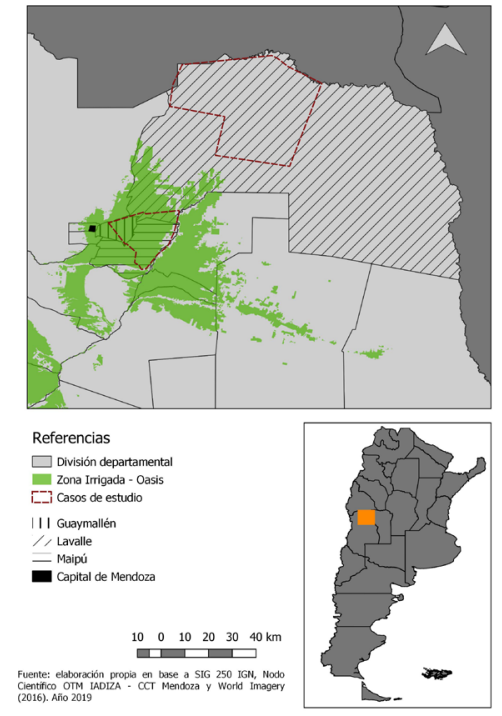

Figura 1: Norte de la provincia de Mendoza donde se señalan las zonas de estudio

Fuente: Elaboración propia en base a SIG 250 IGN. Nodo científico OTM IADIZA - CCT Mendoza y Word Imagery 2016.
Para identificar a las unidades de análisis se realizaron salidas al campo donde se comenzó a trabajar con las unidades que se encontraban en cercanía a las rutas principales, y a partir de allí, mediante un muestreo por bola de nieve, pudimos ubicar a las viviendas construidas con quincha a la vez que los actores relevantes en cuanto al manejo de esta técnica constructiva. En la zona no irrigada se identificaron un total de 12 viviendas donde se utiliza la quincha, mientras que en la zona irrigada se identificaron un total de 30 viviendas construidas con esta técnica constructiva.

Una vez reconocidas las viviendas, se aplicaron las técnicas de observación directa y de la entrevista en profundidad. Mediante la observación directa se reunieron datos en relación a los tipos de materiales y técnicas utilizadas en la vivienda y en la ejecución de los muros de quincha. Las entrevistas en profundidad (Taylor y Bogdan, 1992) nos permitieron, a través de una guía de preguntas abiertas, obtener datos respecto de los materiales naturales disponibles en el sitio, la mano de obra empleada en la construcción de la vivienda, el tiempo de mantenimiento que demanda la quincha, las actividades productivas que se realizan y sobre las prácticas socioculturales. Las entrevistas se aplicaron en un primer momento a los pobladores relevantes reconocidos en las comunidades en cuanto al armado del muro de quincha $y$, en un segundo momento, procedimos a contactar a otros pobladores. Luego, se realizó el análisis de la información mediante el análisis de contenido, con la finalidad de comparar los datos obtenidos y asegurando una respuesta al problema de investigación.

\section{Las técnicas de construc- ción con tierra y sus deriva- dos}

La tierra, como material de construcción, se encuentra presente en la superficie terrestre en diferentes composiciones, lo que permite que pueda ser procesada de varias maneras. El término "barro" es la forma en que se denomina comúnmente a la tierra arcillosa, presentando diferentes nombres según la técnica constructiva, lo cual es un reflejo de la localización y de la cultura en donde se construye. Técnicamente se reconocen cuatro componentes sólidos básicos de la tierra: arcilla, grava, arena y limo. La arcilla es responsable de otorgar la cohesión al barro al secarse y endurecer, cuyos minerales son los responsables de la mayor o menor resistencia de la misma, pero es un elemento inestable frente al agua y la humedad. El resto de los componentes, grava, arena y limo responden al grupo de los materiales inertes que forman parte del esqueleto granular resistente del barro, cohesionados todos ellos por la arcilla.

Los tres grandes grupos que desarrollan los fundamentos de este tipo de construcción son: tierra apisonada o tapia, adobe y técnicas mixtas de entramados. Sin embargo, existen otros métodos tales como el cob o los bloques de tierra comprimidos (BTC). Es así que se encuentran doce métodos diferentes en donde puede ser empleada la tierra para realizar construcciones (Houben y Guillaud, 
1984; Zhai y Previtali, 2010), lo que ayuda a reforzar el gran alcance de este material en la arquitectura. Este trabajo se va a centrar en las características de las denominadas técnicas mixtas de entramados y específicamente en la quincha.

Las técnicas de entramados reciben diferentes denominaciones de acuerdo a la región geográfica en que se construya, según el material vegetal empleado como esqueleto del muro para sostener el barro, el diseño estructural y, finalmente, según la disposición y separación utilizada entre las cañas, ramas u otro materia que se utilice para el entramado. De acuerdo a estas características constructivas, regionalmente reciben los siguientes nombres genéricos: en Perú, Bolivia y Chile se lo denomina quincha; en Ecuador, Colombia, Venezuela, Panamá y en la mayoría de países centroamericanos, como bahareque; en Cuba se lo conoce como cuje o embarrado, y chuchío en Santa Cruz de la Sierra, Bolivia. También se lo conoce como muro envarillado en el Chaco boliviano; enquinchado o palo a pique en Brasil; muros entramados o emparrillados en España y torchis en Francia. En las regiones argentinas del Noroeste y de Cuyo (centro-oeste del país), genéricamente la técnica mixta es conocida como quincha y en la región Noreste de Argentina, se denomina enchorizado, estanteo o palo a pique.

El sistema constructivo de quincha prefabricada se caracteriza, principalmente, por ser utilizada para la conformación de los cerramientos de las viviendas. Actualmente la quincha prefabricada está adquiriendo relevancia, debido a que sus características principales son el bajo peso de los muros y su flexibilidad, que benefician el comportamiento frente al sismo, en comparación con otros sistemas como el adobe o la tapia (Blondet, Vargas, Tarque e Iwaki, 2011). Simultáneamente el reducido espesor de muro, e $=0.10 \mathrm{~m}$, y su favorable respuesta térmica, permite a los muros resguardar los ambientes interiores del calor durante el día y entregarlo al ambiente interior en el transcurso de la noche, durante las bajas temperaturas exteriores, convirtiendo a las construcciones de quincha en una opción habitaciona apta para ser desarrollada en diversas localidades. E sistema consiste básicamente en una estructura portante de madera y los muros son construidos con bastidores de madera aserrada. En el interior llevan un entramado de cañas de Castilla (Arundo donax) y luego lleva un relleno de barro rico en arcilla mezclado con paja. Las terminaciones son con revoques de arcilla o a la cal (Cuitiño, Esteves, Maldonado y Rotondaro, 2015).

\section{Oferta ambiental y cultura en} la construcción de la vivienda vernácula en el norte de Mendoza

A continuación, se presentan los resultados vinculados al estudio de la quincha y sus variaciones según la zona de emplazamiento. Para ello se comienza exponiendo las características naturales de cada caso de estudio respecto a la disponibilidad de agua, tipo de suelo, vegetación y características socioeconómicas. Luego se realiza la descripción del sistema de la quincha y la mano de obra utilizada en la construcción.

\subsection{La quincha en tierras secas no irrigadas de Mendoza}

Los distritos de Lagunas del Rosario y Asunción se encuentran en la parte baja de la cuenca del Río Mendoza, dentro de las zonas no irrigadas del departamento de Lavalle. En este sitio se ubican algunos de los lugares más áridos de la Argentina, de agrestes condiciones climáticas (Abraham y Prieto, 1999). Anualmente, en esta zona, se registran entre $80 \mathrm{~mm}$ a $150 \mathrm{~mm}$ de lluvia, y los suelos son arenosos con fuertes concentraciones de arcilla en los bajos (Chiavazza, 2010).

En relación con la vegetación, el sector se localiza dentro de la Provincia Fitogeográfica del Monte. La única especie que llega a formar bosques aislados es el algarrobo (Prosopis Flexuosa) acompañado por otros árboles como el chañar (Geoffroea decorticans) y el retamo (Bulnesia retama). Entre los pastos, sobresalen Junquillo (Sporobolus rigens) y Coirón (Stipa sp.) (Soria, Salomón, Rubio y Fernández, 2007). Actualmente, el bosque nativo se encuentra diezmado a causa del excesivo aprovechamiento que tuvo lugar a finales del siglo XIX y principios del siglo XX. En sintonía, la disponibilidad de agua superficial en la zona es prácticamente nula debido a la mayor apropiación del caudal del río en la parte media de la cuenca, donde se asienta la ciudad y el oasis. La tala indiscriminada del bosque nativo sumado a la falta de agua desencadenó, desde mediados del siglo $X X$, graves procesos de desertificación, vigentes en toda la zona (Abraham y Prieto, 1999).

Respecto a las características socioeconómicas, en las tierras secas no irrigadas de Lavalle se desarrollan economías de subsistencia, donde la actividad productiva principal es la cría de ganado caprino a escala familiar, aunque también se registran en menor medida otras actividades como la venta de guano y la realización de artesanías con cuero, arcilla y madera.

En la construcción de las viviendas predomina el uso de barro sin cocer, donde sobresale el uso del adobe y en menor medida de la quincha y otros materiales industrializados, como el ladrillo cocido. Las viviendas en las cuales se utiliza quincha (en toda la envolvente o de forma parcial) se resuelve en todos los casos de la misma manera. La estructura principal se conforma por el uso de algarrobo o chañar en los postes principales y vigas (Fig. 2, punto 1). Luego, se agrega un entramado horizontal o vertical hecho con caña de Castilla (Arundo donax) que se vincula a la estructura principal mediante alambre (Fig. 2, punto 2). A lo largo del muro se suelen colocar postes de menor diámetro o cañas en sentido contrario para aumentar la rigidez del sistema (Figura 2, punto 3). Finalmente, sobre la caña se aplica una mezcla de barro y agua a la que se agrega actualmente guano de caballo para adicionar fibras y también para otorgar cierta elasticidad y evitar fisuras durante el proceso de secado. Este dato resulta importante, ya que anteriormente se colocaba paja de trigo, cultivo que se realizaba en el lugar, pero con la merma en la disponibilidad de agua del río para su producción, fue imposible continuar con este cultivo. A esto se suma la dificultad que encuentran los pobladores para conseguir paja de trigo en el mercado, 
y por ello utilizan el guano de caballo que es el material que disponen en el medio.

La estructura no posee cimientos y solo los postes verticales van enterrados, apoyándose el muro directamente sobre el suelo (Figura 2, punto 4), provocando en algunos casos problemas de humedad debido al ascenso capilar del agua por los muros de tierra. Para proteger al muro de la acción del agua de lluvia se construyó un alero (Figura 2, punto 5). Pero este alero no es continuo en toda la envolvente, y por ello algunas paredes quedan al descubierto y presentan mayor desgaste, lo que se traduce en mayores tareas de mantenimiento (Figura 2, punto 6). Ciertamente, la construcción con techo plano en toda la zona (correas de madera, entramado de caña y finalmente torta de barro) incide en la dificultad para realizar aleros en ciertos muros.

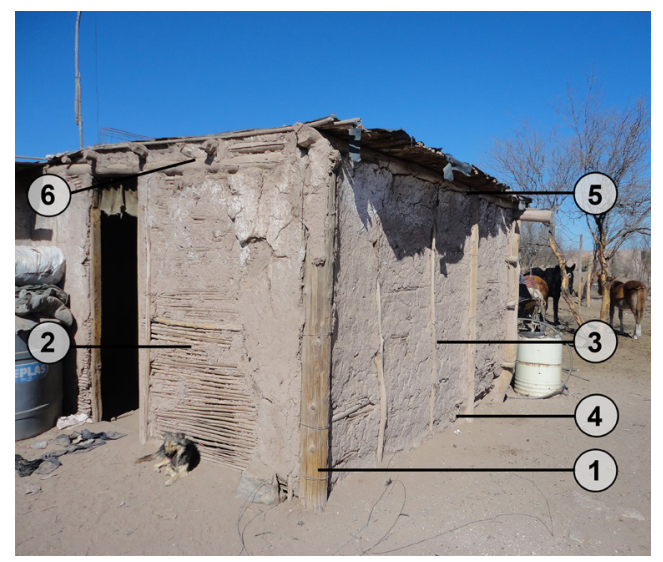

Figura 2: Puesto en Lagunas del Rosario, Lavalle, con e sistema constructivo de la quincha y sus componentes. Fuente: Propia.

A partir de las entrevistas realizadas a los habitantes, se pudo tomar conocimiento de que la quincha ya no es un material de uso corriente en esta zona por el espesor final que alcanza la pared (aproximadamente 0,10m) pero principalmente por la falta de fibras naturales adecuadas para el armado de la mezcla del barro, ya que se desprende con mayor facilidad. Viñuales (2007) se refiere en estas dos problemáticas como las desventajas del sistema de entramado, haciendo referencia a la fragilidad del conjunto por el espesor final que alcanza y la contracción del secado según los materiales empleados. Por ello, actualmente los pobladores prefieren utilizar adobe, por el espesor que posee (aprox. 0,20m). No obstante, en muchos casos el sistema de la quincha se utiliza debido a la rapidez del armado del muro, sumado a que muchas veces se trata de paredes provisorias, que luego los pobladores reemplazan por muros de adobe.

La mano de obra empleada para la construcción de los muros de quincha es local, donde interviene la familia, los vecinos y los que estén dispuestos a colaborar. En esta tarea es donde se transfieren los conocimientos de los adultos a los jóvenes respecto a las formas constructivas y el manejo de los materiales naturales. Por ello, todas las viviendas de quincha en la zona se resuelven de la misma manera. Esta similitud implica que los pobladores manejan los mismos marcos compositivos, producto de la cultura y de sus prácticas arraigadas en la comunidad (Figura 3).
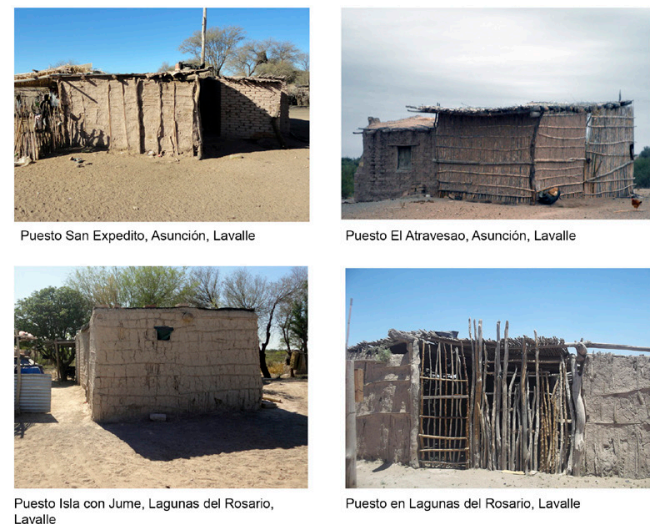

Puesto El Atravesao, Asunción, Lavall
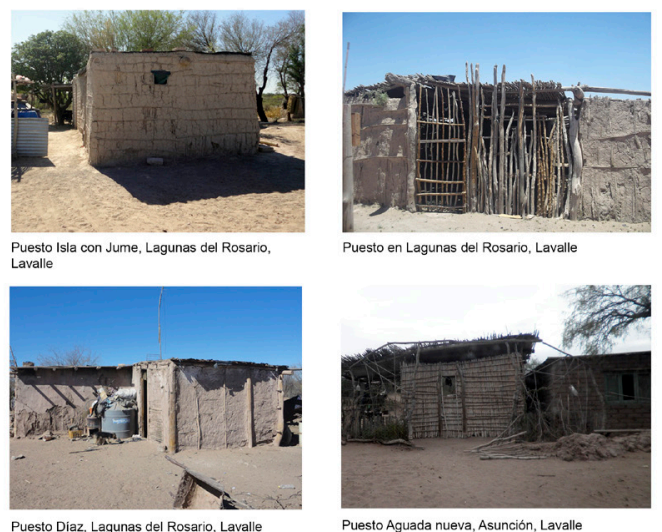

Puesto Diaz, Lagunas del Rosario, Lavalle

Puesto Aguada nueva, Asunción, Lava

Figura 3: Diferentes viviendas donde se observa la resolución constructiva de la quincha en tierras secas no irrigadas

Fuente: Propia.

\subsection{La quincha en tierras secas irrigadas -oasis-}

El Cinturón Verde de Mendoza es un territorio agrícola orientado principalmente hacia la producción hortícola. Esta zona se emplaza en la parte media de la cuenca del Río Mendoza y abarca parte de los departamentos de Guaymallén y Maipú. La agricultura en general fue favorecida en esta zona por la mayor disponibilidad de agua superficial, ya que las precipitaciones no superan los $250 \mathrm{~mm}$ anuales. La actividad agrícola de la zona ha desplazado a la vegetación autóctona, donde los árboles que se encuentran en el sitio se corresponden con aquellos implantados por la sociedad, aprovechando la disponibilidad de agua superficial y generando un microclima respecto del clima árido para disponer de sombra. Algunas de las especies que predominan en este sector son álamos (populus nigra) y sauces (Salix humboldtiana). Entre los arbustos aprovechables para mezclar con el barro de relleno se encuentran la cortadera (Cortaderia sp.) y la totora (Typha dominguensis), que algunos pobladores cultivan en sus terrenos para abastecimiento propio o para la venta. Los suelos son ricos en materia orgánica con fuerte tendencia a la salinización, que responde a la presencia de las capas freáticas cerca de la superficie y material arcilloso que retarda la absorción del agua de riego, lo que incide en la mayor presencia de humedad en el suelo, aunque la presencia de suelos arcillosos es favorable para su uso en la construcción natural.

En esta zona del Cinturón Verde de Mendoza se encuentran edificaciones, principalmente viviendas y galpones vinculados a las actividades agrícolas, materializadas con el sistema constructivo de la quincha. En este caso, se observan adecuaciones respecto al 
sistema constructivo de las tierras secas no irrigadas, que responden a la mayor presencia de agua y humedad en e suelo, a la vez que se aprecia un mayor uso de materiales industrializados, como chapa acanalada de zinc en las cubiertas.

El sistema constructivo tradicional del lugar presenta un cimiento materializado en la mayoría de los casos con piedra tosca, disponible en el lugar. En algunas construcciones se encontró un sobrecimiento realizado con el mismo material o recubierto con hormigón, cuya función es evitar que el muro de barro entre en contacto con el agua o la humedad del suelo (Figura 4, punto 1). La estructura portante se conforma con columnas y vigas de madera, a la cuales se agrega un poste en diagona para rigidizar la estructura (Figura 4, punto 2). En todos los casos relevados, estos elementos no son utilizados de forma natural, como se extrae en el medio, ya que por su sección cuadrada se deduce que han sido previamente trabajados para lograr esta uniformidad.

Luego, se encuentra un entramado que en algunos casos es de caña de Castilla, y en otros casos son listones de madera de álamo o tablas de sección rectangular que se colocan de forma perpendicular a la estructura portante (Figura 4, punto 3). Esta última es la más utilizada porque facilita su unión con el poste vertical, ya que se disponen los listones de forma horizontal y se clavan en los postes principales tanto del lado interior como del exterior dejando un vacío entre estos entramados, el cual se rellena luego con una mezcla de barro. Finalmente, el muro se revoca con barro o con hormigón en ambas caras, alcanzando un espesor final de aproximadamente $0,20 m$ a $0,25 m$ (Figura 4, punto 4).

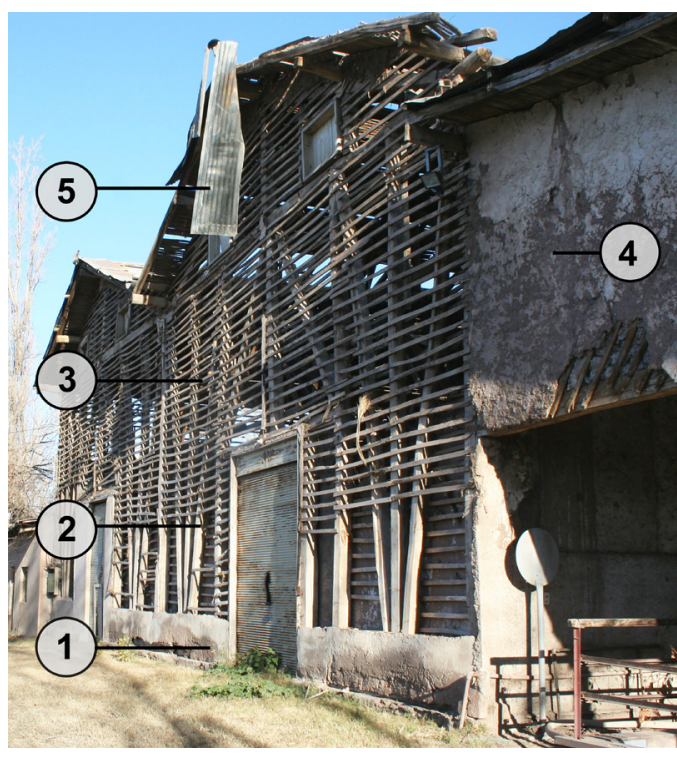

Figura 4: Vivienda y bodega donde se observa el sistema de la quincha en Guaymallén y sus componentes.

Fuente: Propia.

En la zona de análisis, las viviendas presentan techo a dos aguas, lo que facilita la disposición de aleros en toda la envolvente para proteger a los muros de la acción de la lluvia. El techo se materializa con correas de madera, entramado de caña o tablas de madera, una capa de barro y finalmente chapa acanalada de zinc, o teja cerámica (Figura 4, punto 5).

La mano de obra es local, permitiendo la autoconstrucción, ya que es la misma familia la que se encarga de la construcción. Por esta misma razón, se trata de una forma constructiva enraizada en las costumbres de la población de la zona, y por ello, se encuentra un patrón constructivo que demuestra su amplio uso (Figura 5).
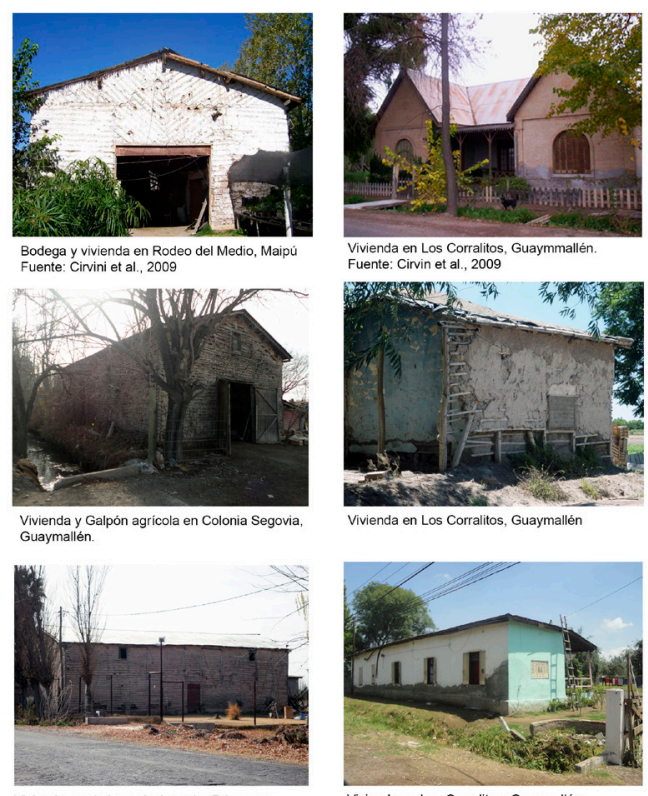

Vivienda y ga tron
Guaymallén

Figura 5: Fotografías donde se muestra el patrón constructivo del sistema de la quincha en la zona del Cinturón Verde de Mendoza.

Fuente: Propia y Cirvini et al., 2009

\section{Discusiones}

Mediante el estudio del sistema de la quincha en diferentes escenarios rurales del norte de Mendoza, se observa que presenta algunas variaciones en la forma en la que se materializa el muro, ya que en las tierras secas no irrigadas se trata de un entramado horizontal o vertical de cañas a la que se agrega el revoque interior y exterior. En la zona del oasis, en cambio, los entramados contienen un relleno de barro, además del revoque, asegurando de esta manera una pared más sólida y disminuyendo huecos producto del desmoronamiento del revoque (Figura 6). Además, la mayor presencia de madera en la zona irrigada permite la conformación de una estructura más rígida, con postes diagonales.

Otra de las ventajas que presenta el sistema en zonas irrigadas es la colocación de un sobrecimiento de piedra tosca, que ayuda a prevenir el contacto del muro de barro con la humedad del suelo o el agua. Esto también resulta posible por la disponibilidad de piedra en el sitio, ya que en zonas no irrigadas no es común encontrar este material. De esta forma, se logra disminuir la acción de la humedad del suelo que afecta al sistema. De la misma manera, la presencia de aleros en toda la envolvente 
resulta adecuada en zonas irrigadas, ya que evita que el agua de lluvia entre en contacto con el muro de barro, afectando su resistencia.

Queda de manifiesto que las características del sistema, así como la forma de materializarlo, responde a los bienes naturales disponibles. Sin embargo, también influye la cultura de la población local. En el caso de las zonas irrigadas, la forma de ejecutar el sistema de la quincha responde, de acuerdo a trabajos de autores como Prieto, Rojas, Castrillejo y Hernández (2012), y Avellaneda, Crocco y Estevez ${ }^{1}$ (2017), a la inmigración, haciendo referencia a la presencia de pobladores de origen francés y mallorquín que se establecieron en la zona a principios del siglo XX y que serían quienes introdujeron las variaciones al sistema de la quincha de acuerdo a manejo de la técnica en sus lugares de origen. Esto se refuerza en las entrevistas a los pobladores de avanzada edad, quienes contaron sobre la masiva presencia de inmigrantes en el Cinturón Verde.

En la zona no irrigada, los actores sociales entrevistados comentaron que la utilización de ciertos materiales no solo tiene que ver con las posibilidades que el entorno natural les brinda sino también con una identidad simbólica asociada a los conocimientos acumulados

\section{QUINCHA EN ZONAS NO IRRIGADAS}

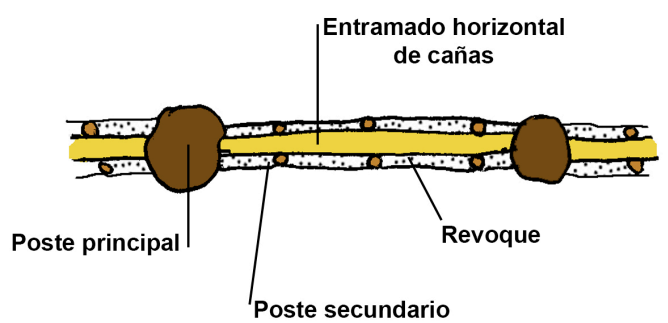

QUINCHA EN ZONAS IRRIGADAS

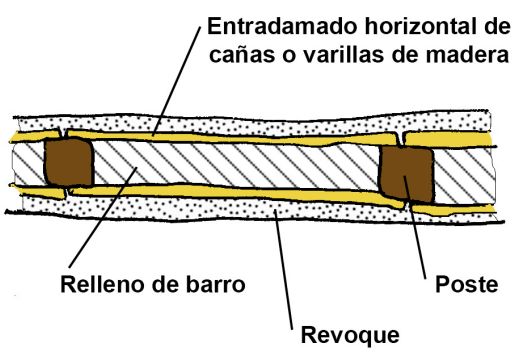

Figura 6: Esquemas en planta del sistema de la quincha en cada caso analizado.

Fuente: Propia.

${ }^{1}$ Avellaneda et al. analizan las viviendas construidas con quincha en la localidad de Médano de Oro, provincia de San Juan, que se encuentra al norte de Mendoza. Por las descripciones de la autora, se trata de mismo sistema constructivo que en la zona del Cinturón Verde de Mendoza, aspecto desde el que se infiere que se trata de inmigrantes oriundos del mismo sitio con los mismos conocimientos respecto a la construcción con barro. a través del tiempo. Aquí radica la importancia de la arquitectura vernácula como patrimonio material pero que permite identificar aspectos intangibles, como las señas de identidad de una comunidad y los conocimientos tradicionales.

Identificar las características del sistema constructivo de la quincha en ambos casos nos permite comprender cómo los pobladores resuelven su necesidad de vivienda con los materiales de que disponen en el medio. Esto se presenta como un hallazgo para desalentar las actuaciones que tiendan a considerar al sistema de la quincha como homogéneo en todo el territorio provincial. Por el contrario, prestando atención a los materiales utilizados y la forma en que se construye, se podría apoyar a las comunidades para la conservación y puesta en valor de su arquitectura, con sus características propias.

En la introducción se exponía que la construcción con tierra cruda se asoció al atraso y la pobreza, lo que favoreció la desestimación de la construcción con este material, relegando también el reconocimiento de la dimensión patrimonial de esta arquitectura. A su vez, en Mendoza se suma la presencia de sismos, lo que también incidió en la desestimación de las construcciones con tierra. No obstante, se observa que el sistema de entramados presenta una buena respuesta a los movimientos sísmicos. A todo ello hay que sumar que la construcción con materiales naturales del sitio se presenta como una estrategia para hacer frente al déficit habitacional y reducir costos en la construcción. La mano de obra local también permitiría reducir costos a la vez que generar mayor apropiación de la vivienda y del sistema constructivo. No obstante estas ventajas factibles de ser aprovechadas, nos interesa remarcar que la mayor parte de las viviendas que fueron relevadas presentan deficiencias en la forma en que utilizan los materiales, así como patologías derivadas de la falta de aleros, de sobrecimientos, problemas de humedad y falta de cimientos adecuados, entre otras. Estos problemas muchas veces derivan de las adaptaciones que los pobladores realizan al sistema con los materiales naturales que tienen a mano. Por ello, hay que considerar que la producción de conocimientos respecto a la edificación con tierra encuentra importantes avances en el ámbito académico y científico, y que su consideración resulta clave para asegurar la calidad constructiva de esta arquitectura.

La presencia de arquitectura vernácula en quincha en Mendoza, con sus variaciones, se presenta como un hallazgo para repensar el rol de esta arquitectura en las zonas rurales, fuertemente anclada a las comunidades. Queda pendiente de respuesta la visión y acciones que ejercen las administraciones públicas municipales respecto a la arquitectura en tierra en las zonas rurales, para poder establecer lineamientos factibles de ser empleados en la gestión y valorización de lo vernáculo como patrimonio. 


\section{Conclusiones}

El sistema constructivo de la quincha presenta variaciones en diferentes sectores del norte de Mendoza. Estas variaciones se vinculan con la disponibilidad de materiales naturales en el sitio y por la cultura de la población local. Las diferencias del sistema constructivo de la quincha en cada caso enriquecen los matices que presenta la arquitectura vernácula rural en la provincia, ampliando su importancia. Ciertamente, a partir de conocimiento de las características que presenta e sistema resulta factible poner en valor a esta arquitectura y las acciones necesarias para su conservación.

La mano de obra local es un aspecto clave, ya que se encuentran conocimientos tradicionales y técnicas de construcción adaptadas a la cultura y a la disponibilidad de bienes naturales. Por ello, se hace referencia a un patrimonio vivo que se encuentra vigente. Considerar articular los conocimientos tradicionales de los pobladores con los avances técnicos y científicos permitiría alcanzar la perdurabilidad de los sistemas constructivos vernaculares de la quincha, que atienda a temas de identidad local combinado con los requerimientos técnicos de calidad constructiva de las épocas actuales. Se trata de considerar el dinamismo y transformaciones de lo vernáculo asociado a los conocimientos científicos que permitan su conservación y uso en el tiempo.

\section{Agradecimientos}

Los autores agradecen a la Universidad de Mendoza por financiar esta investigación y la Arq. Romina Sales por la diagramación de las figuras.

Cómo citar este artículo/How to cite this article: Esteves, M. y Cuitiño, G. (2020). El sistema constructivo de la quincha en zonas rurales del Norte de Mendoza (Argentina). Estoa. Revista de la Facultad de Arquitectura y Urbanismo de la Universidad de Cuenca, 9(17), 93-102. doi: 10.18537/ est.v009.n017.a08 


\section{Referencias bibliográficas}

Abraham, E. M. y Prieto, R. (1999). Guanacache, la travesía de los profundos cambios. En R. Gotthelf (Dir.), Guanacache, Fidel Roig Matons, pintor del desierto (pp. 107-125). Mendoza, Argentina: EDIUNC.

Avellaneda, A, Crocco, E. y Estévez, F. (2017). Vivir y trabajar la chacra: la vivienda de quincha de los pequeños productores del Médano de Oro. Revista Kairos, (12) Recuperado de https://www.revistakairos.org/vivir-ytrabajar-en-la-chacra/

Blondet, M., Vargas, J., Tarque, N. e Iwaki, C. (2011) Construcción sismorresistente en tierra: la gran experiencia contemporánea de la Pontificia Universidad Católica del Perú. Informes de la construcción, 63 (523), 41-50. Recuperado de http://informesdelaconstruccion. revistas.csic.es/index.php/informesdelaconstruccion/ article/view/1251

Canivell, J. y Pastor, G. (2018). Evaluación de la arquitectura vernácula construida en tierra en la provincia de Mendoza. Planteamientos y resultados. Arquitectura, ciudad y entorno, 12 (37), 133-154. doi:10.5821/ace.13.37.5180

Chiavazza, H. (2010). Ocupaciones en antiguos ambientes de humedal de las tierras bajas del norte de Mendoza: sitio Tulumaya (PA70). Intersecciones en antropología, 11 (1), 41- 57 .

Cirvini, S. y Manzini, L. (2016). Diagnóstico y evaluación de las viviendas en el patrimonio arquitectónico de Mendoza, Argentina. Apuntes, 29 (1), 44-59. Recuperado de https://revistas.javeriana.edu.co/index.php/ revApuntesArq/article/view/18384

Cirvini, S. (2017). Vernacular heritage in Mendoza (Cuyo region, Argentina). En: C. Mileto, F. Vegas, L. García Soriano y V. Cristini (Eds). Vernacular and Earthen Architecture: Conservation and Sustainability (Capítulo 11). Londres, Reino Unido: CRC Press.

Cirvini, S., Gómez Voltán, J., Manizini, L., Raffa, C., Angeleri, F. y Marchionni, F. (2009). Patrimonio arquitectónico del Área Metropolitana de Mendoza. Métodos y técnicas para su detección, catalogación y evaluación como recurso. Mendoza, Argentina: CONICET.

Cortés Pedrosa, J. (2013). La arquitectura popular como modelo de edificación sostenible. El ejemplo de Tierra de Campos. Observatorio Medioambiental, 16, 185-206.

Cuitiño, G., Esteves, A., Maldonado, G., y Rotondaro, R. (2015). Analysis of thermal transmittance and resistance to soft shock in wattle walls. Informes de La Construcción, 67(537). doi: 10.3989/ic.12.082

Grosso Cepparo, V. (2017). Agua y tierras secas. Lecturas críticas sobre la escasez hídrica en el departamento de Lavalle (Mendoza, Argentina). Estudios Socioterritoriales, (22), 27-45. Recuperado de http://www.scielo.org.ar/ pdf/esso/v22/v22a03.pdf
Houben, H. y Guillaud, H. (1984). Earth construction promer: project, earth construction technologies appropriate to developing countries. Lovaina, Bélgica: Centre de Recherches Architecturales.

ICOMOS (1999). Carta del patrimonio vernáculo construido. México D.F., México. Recuperado de http:// www.international.icomos.org/charters/vernacular sp.pdf

Lacoste, P., Premat, E. y Bulo, V. (2014). Tierra cruda y formas de habitar en el Reino de Chile. Revista Universum, 1 (29), 85-106.

Lárraga Lara, R., Aguilar Robledo, M., Reyes Hernández, H. y Fortanelli Martínez, J. (2014). La sostenibilidad de la vivienda tradicional: una revisión del estado de la cuestión en el mundo. Revista de arquitectura, 16 (1), 126-133. Recuperado de https://editorial.ucatolica.edu. co/index.php/RevArq/article/view/65

Montaña, E., Torres, L., Abraham, E., Torres, E. y Pastor G. (2005). Los espacios invisibles. Subordinación, marginalidad y exclusión de los territorios no irrigados en las tierras secas de Mendoza, Argentina. Región y sociedad, 17 (32), 3-32.

Prieto, R., Rojas, F., Castrillejo, T. y Hernández, F. (2012). Procesos ambientales y construcción del territorio a partir de un estudio de caso: La ciénaga del Bermejo, oasis norte de Mendoza 1810-1930. Revista de historia americana y argentina, 47 (2), 175-207.

Rapoport, A. (2003). Cultura, arquitectura y diseño. Barcelona, España: Edicions UPC.

Rotondaro, R. (2018, diciembre, 18). Construir con tierra: tecnología y arquitectura del siglo XX. Notas CPAU. Recuperado de http://www.revistanotas.org/ revistas/41/2295-construir-con-tierra-tecnologia-yarquitectura-del-siglo-xxi

Rufino, J. (2013). Determinación de los problemas técnico-constructivos actuales que afectan la calidad y durabilidad de las viviendas de tierra en la provincia de Uige, Angola. Arquitectura y Urbanismo, XXXIV (2), $27-$ 36. Recuperado de http://rau.cujae.edu.cu/index.php/ revistaau/article/view/253/231

Salman, M. (2018). Sustainability and vernacular architecture: Rethinking what identity is. En: K. Hmood (Ed.), Urban and Architectural Heritage Conservation within Sustainability, Jordania: University of Jordan. doi: 10.5772/intechopen.82025.

Sánchez Quintanar, C. y Jiménez Rojas, E. (2010). La vivienda rural. Su complejidad y estudio desde diversas disciplinas. Luna Azul, (30), 174-196.

Scoones, A. E. (2018). Territorios rurales en Mendoza: inversiones vitivinícolas y avance urbano en el oasis norte. Convergencias, 1 (1), 87-105.

Soria, D., Salomón, M., Rubio, C. y Fernández, R. (2007). Herbario digital secano de Lavalle. Mendoza, Argentina: Laboratorio de Desertificación y Ordenamiento Territorial. 
Recuperado de: https://www.mendoza-conicet.gob.ar/ ladyot/sig-deser/publicac_sig_pdi/trabajos/herbario digital.pdf

Tamayo, J., Malo, G. y García, G. (2019). El dibujo y su aporte a la identificación de valores de la arquitectura vernácula. Estoa. Revista de la Facultad de Arquitectura y Urbanismo de la Universidad de Cuenca, 8 (16), 33-45. Recuperado de https://publicaciones.ucuenca.edu.ec/ ojs/index.php/estoa/article/view/2827

Taylor, S. y Bogdan, R. (1992). Introducción a los métodos cualitativos de investigación. Barcelona, España: Paidós.

Tillería González, J. (2010). La arquitectura sin arquitectos, algunas reflexiones sobre arquitectura vernácula. AUS, (8), 12-15. Recuperado de http://revistas.uach.cl/index. php/aus/article/view/422

Tomasi, J. (2011). Mirando lo vernáculo. Tradiciones disciplinares en el estudio de las "otras arquitecturas" en la Argentina del siglo XX. Área, (17), 68-83.

Torres, L. (2010). Claroscuros del desarrollo sustentable y la lucha contra la desertificación: las racionalidades económicas en el ojo de la tormenta. Estudio de caso con productores caprinos de tierras secas (Mendoza, Argentina). Mundo agrario, 11 (21). Recuperado de https://www.mundoagrario.unlp.edu.ar/article/view/ v11n21a11/374

Urquijo, H. (1972). Tipos predominantes de vivienda natural en la República Argentina. Instituto de Investigaciones de la vivienda. Buenos Aires, Argentina: FAU-UBA-EUDEBA.

Viñuales, G. (2007). Tecnología y construcción con tierra. Apuntes, 20 (2), 220-231. Recuperado de https://revistas. javeriana.edu.co/index.php/revApuntesArq/article/ view/8978

Zhai, Z. y Previtali, J. (2010). Ancient vernacular architecture: characteristics categorization and energy performance evaluation. Energy and buildings, 42 (3), 357-365. 